\title{
Educação Médica e Formação na Perspectiva Ampliada e Multidimensional: Considerações acerca de uma Experiência de Ensino-Aprendizagem
}

Medical Education and Training in the Expanded and Multidimensional Perspective: Considerations about a Teaching-Learning Experience

Claudio José dos Santos Júnior Jailton Rocha Misael Maria Rosa da Silva Valmir de Melo Gomes

\section{PALAVRAS-CHAVE}

- Educação Médica.

- Integração Ensino-Serviço.

- Assistência Integral à Saúde.

- Saúde Pública.
A fragmentação curricular e a separação entre as diversas dimensões da formação médica têm sido responsáveis por incitar o debate sobre a necessidade de lançar mão de estratégias que promovam maior proximidade entre o que se ensina nos cursos de graduação em Medicina e o que, de fato, faz parte do dia a dia dos profissionais de saúde em seus campos de trabalho. Dessa forma, diversas são as propostas de atualização do currículo que vêm sendo discutidas no âmbito das escolas médicas e demais setores envolvidos com a formação profissional. Assim, o presente trabalho tem por objetivo descrever vivências e contribuições das ações realizadas durante o desenvolvimento de dois eixos disciplinares do curso de bacharelado em Medicina da Universidade Estadual de Ciências da Saúde de Alagoas (Uncisal), os quais centram suas propostas em promover a articulação entre ensino, serviço e comunidade, inserindo o discente do primeiro ano do referido curso em cenários do Sistema Único de Saúde (SUS). Quanto à metodologia, trata-se de um trabalho do tipo "relato de experiência", e, para a sua construção, foram empregadas as reflexões dos autores-participantes das disciplinas acerca das ações por eles experimentadas. Utilizando fundamentos advindos da Saúde Coletiva e das ciências humanas e sociais, as experiências pedagógicas e métodos de aprendizagem proporcionados foram capazes de levar os alunos a entenderem, na prática, o funcionamento do SUS e suas dimensões política e administrativa, além de fazerem reflexões acerca dos conceitos de saúde-doença a partir da inserção em vivências práticas na comunidade. Tais atividades configuraram experiências altamente enriquecedoras e espaços privilegiados de formação acadêmica e cidadã para o futuro da atuação profissional em medicina. Ademais, fornecem ferramentas efetivas para a aplicação do que as Diretrizes Curriculares Nacionais preconizam para uma formação médica alinhada às necessidades da comunidade e do SUS. 


\section{KEY-WORDS}

- Medical Education.

- Teaching-Service Integration.

- Integral Assistance.

- Public Health.

\begin{abstract}
Curricular fragmentation, and the separation between the various dimensions of medical education, have led to a debate on the need for strategies to promote a closer alignment between what is taught on undergraduate courses in Medicine and the actual day-to-day work of health professionals in their fields of work. Various proposals for updating the curriculum are currently being discussed in medical schools and other sectors involved with vocational training. The aim of this study is to describe the experiences and contributions of actions carried out during the development of two disciplinary areas of the Bachelor of Medicine Course at Universidade Estadual de Ciências da Saúde de Alagoas (Uncisal). These proposals are focused on promoting articulation between teaching, service, and the community, giving first year students of the course practical experience of various scenarios of the Unified Health System (Sistema Único de Saúde - SUS). In terms of methodology, this work is a "Report of Experience". For its construction, it uses the reflections of the authors-participants of the disciplines on their experiences. Using the foundations of Collective Health and Human and Social Sciences, the pedagogical experiences and learning methods provided enabled the students to understand, in practice, how the Unified Health System works, and its political and administrative dimensions, as well as reflecting on the concepts of health-disease based on their practical experiences in the community. These activities have shaped highly enriching experiences and privileged spaces of academic and formation of citizens for the future of professional practice in medicine. They also provide effective tools for the application of the recommendations of the National Curricular Guidelines for medical education that is aligned with the needs of the community and of the SUS.
\end{abstract}

Recebido em: 10/8/2018

Aceito em: 3/9/2018

\section{INTRODUÇÃO}

Um dos maiores desafios com que a medicina se depara nos dias atuais diz respeito à necessidade de atualização do processo de formação de seus estudantes. Para alguns autores, essa necessidade se dá pela realidade de dissociação e inadequação entre os conteúdos abordados no universo acadêmico e as necessidades e demandas comunitárias ${ }^{1}$.

A fragmentação curricular e a separação entre as diversas dimensões da formação médica têm sido responsáveis por incitar o debate sobre a necessidade de lançar mão de estratégias que promovam maior proximidade entre o que se ensina nos cursos de graduação em Medicina e o que faz parte do dia a dia dos profissionais de saúde em seu campo de trabalho ${ }^{2}$.

Franco et al. ${ }^{3}$ indicam que, devido a essa realidade, há uma tendência mundial de edição das diretrizes de formação dos cursos médicos com vistas à reformulação e à inserção de habilidades e competências consideradas essenciais ao profissional da medicina para atuar em distintos cenários e circunstâncias, reconhecendo as especificidades das comunidades e fazendo associações entre a saúde das populações e o contexto em que estão inseridas.

Uma das propostas para promover essa adequação é a de introduzir, desde o início, e perpassando toda a forma- ção acadêmica, elementos fundamentais que devem reger a conduta do profissional de saúde e seu relacionamento com a sociedade ${ }^{4}$. Alguns autores enfatizam que, nesse ponto, há necessidade de uma abordagem pautada na humanização, na integralidade e nos preceitos éticos e de responsabilidade social, destacando tais aspectos como estratégicos para o melhor exercício da profissão ${ }^{5}$. Outros afirmam que o acolhimento e a empatia, quando presentes no dia a dia dos profissionais, possibilitam maior adequação e resolutividade da atuação profissional quanto às necessidades das comunidades atendidas ${ }^{6}$.

Lima et al..$^{5}$ reconhecem que a formação de recursos humanos qualificados para atuar tanto na prevenção de agravos quanto na promoção de qualidade de vida da população se torna uma questão urgente no Brasil. Para os autores, o fato de o País ser uma nação plural e de grande extensão territorial, além de possuir um sistema público de saúde universal, é um indício que representa enormes desafios para a consolidação e o atendimento dos princípios de equidade e universalidade previstos na criação do Sistema Único de Saúde (SUS). Os pesquisadores defendem a implantação de uma carga horária exclusiva no currículo universitário de atividades que conduzam à compreensão do desafio de consolidar um atendimento com integralidade, por ser essa uma ferramenta ímpar para subsidiar a promoção 
da saúde e os pilares do SUS, além de valorizar a pactuação e a efetivação de projetos político-pedagógicos que propiciam saberes sob a ótica da aproximação com grupos sociais.

À luz dos avanços na área de educação médica, várias são as correntes teóricas que defendem experiências pautadas em novos cenários e que incitam o debate sobre a necessidade de lançar mão de estratégias capazes de promover maior proximidade entre o que se ensina nos cursos de Medicina e o que efetivamente se faz no dia a dia do profissional nos diversos campos de trabalho, principalmente no âmbito do SUS, de modo a dar condições ao futuro profissional de compreender as dificuldades e possibilidades da sua atuação e a dar subsídios para que ele possa buscar estratégias para superar tais situações, desenvolvendo seu trabalho e atendendo às demandas sociais por saúde $e^{7,8}$.

Diante desse cenário, o presente trabalho se propõe a relatar as vivências e possibilitar reflexões acerca do contributo das ações realizadas durante o desenvolvimento de dois eixos/módulos disciplinares do curso de graduação em Medicina de uma instituição de ensino do Estado de Alagoas. Notadamente, a experiência resultou em atividades de ensino-aprendizagem altamente enriquecedoras para os acadêmicos durante todo o desenvolvimento do estudo de tópicos relativos à saúde coletiva e temas correlatos.

\section{METODOLOGIA}

Trata-se de um relato de experiência dos trabalhos desenvolvidos ao longo dos módulos integradores "O Médico, o Indivíduo e a Comunidade I" (MIC I) e "O Médico e o Seu Trabalho I" (MST I), do curso de bacharelado em Medicina da Universidade Estadual de Ciências da Saúde de Alagoas (Uncisal). Ambos os módulos são ministrados por uma equipe multidisciplinar que conta com profissionais da área de saúde e docentes com formação nas áreas de ciências humanas e sociais.

A ementa básica do módulo de MIC I versa sobre o processo de saúde-doença, resgatando seus fatores determinantes e condicionantes, sobretudo no SUS, as políticas de saúde ao longo da história e os modelos assistenciais vigentes; o módulo de MST I aborda as tecnologias em saúde, a formação médica e suas diretrizes, direitos humanos, movimentos sociais, políticas públicas afirmativas e complementares em saúde e princípios de equidade, igualdade e alteridade nas relações interprofissionais e com os usuários.

Desenvolvidas durante o ano letivo de 2016, as disciplinas contaram com atividades teóricas e com práticas na comunidade. As atividades teóricas tiveram apoio de diversos recursos educacionais, a exemplo de documentários, artigos, seminários temáticos, filmes, dinâmicas e debates direcionados.
As atividades práticas de ambas as matérias foram realizadas em Unidades Básicas de Saúde (UBS) de Maceió, respectivamente, do primeiro e do quinto distrito sanitário e adjacências. Tais atividades incluíram a observação da dinâmica de trabalho dessas unidades, o acompanhamento de agentes de saúde em suas tarefas junto à comunidade e a participação em ações educativas desenvolvidas com usuários e profissionais da Estratégia de Saúde da Família (ESF) e do Núcleo de Apoio à Saúde da Família (Nasf), além de, no caso específico de MST I, visita a comunidades indígenas, terreiros de religião de matriz afro-brasileira e espaços de promoção de saúde e ativismo organizados pelo público LGBTT (Lésbicas, Gays, Bissexuais, Travestis e Transexuais).

Os textos basilares para as aulas teóricas de MIC I foram as obras "Tratado de saúde coletiva", da Editora Revista e Aumentada, e "O que é o SUS?", da Editora Fiocruz, além das Leis 8.080/1990 e 8.142/1990. Em MST I, a cada aula, um novo artigo relacionado ao conteúdo da semana era apresentado. Na etapa de abordagem das políticas públicas afirmativas, foram enfatizadas as Políticas Nacionais de Saúde Integral LGBTT, da População Negra e dos Povos Indígenas, além do Caderno de Educação Popular e Saúde, ambos os documentos de autoria do Ministério da Saúde.

A metodologia adotada para o desenvolvimento do presente trabalho foi a de pesquisa-ação com abordagem qualitativa, sendo que em sua construção foram empregadas as reflexões dos próprios autores (discentes e professores das disciplinas) acerca das ações por eles experimentadas e utilizados registros escritos realizados durante as atividades teórico-práticas das disciplinas.

O referencial teórico utilizado na discussão foi obtido por meio de pesquisa bibliográfica em artigos científicos indexados nas bases de dados Scientific Electronic Library Online (SciELO) e Google Acadêmico sobre as temáticas "educação médica", "integração ensino-serviço", "assistência integral à saúde" e "Sistema Único de Saúde".

De acordo com as orientações dos Periódicos Eletrônicos de Psicologia ${ }^{9}$, o gênero textual relato de experiência possui relevância devido a sua pertinência, à importância dos problemas nele expostos e, em função do nível de generalização, à aplicação de procedimentos ou de resultados de intervenções em outras situações similares, ou seja, serve como colaboração à práxis metodológica de uma área do saber.

Segundo $\mathrm{Gil}^{10}$, a pesquisa-ação tem base empírica, apoia-se nas experiências vividas e na observação dos pesquisadores e geralmente está relacionada à solução, de forma coletiva e participativa, de um problema em que os pesquisadores estão envolvidos diretamente e de modo cooperativo ou partici- 
pativo. Para Kemmis e McTaggart ${ }^{11}$, trata-se de uma forma de investigação baseada em uma autorreflexão coletiva empreendida pelos integrantes de um grupo de maneira a melhorar a racionalidade e a justiça de suas próprias práticas sociais e educacionais, como também seu entendimento dessas práticas e de situações onde estas acontecem.

Por se tratar de uma experiência de ensino-aprendizagem, não houve necessidade de formalização do procedimento por meio de TCLE e/ou da apreciação por Comitê de Ética em Pesquisa, nos moldes da Resolução nº 510/2016 do Conselho Nacional de Saúde.

\section{RESULTADOS E DISCUSSÃO}

Os módulos de MIC I e MST I surgiram a partir da necessidade de implementar, no âmbito do projeto político-pedagógico do curso de Medicina da Uncisal (PPC), disciplinas que permitissem a articulação entre teoria e prática e possibilitassem aos estudantes, desde cedo, o contato com a realidade do SUS, da atenção primária e da influência dos contextos social, econômico e cultural no âmbito da saúde, em atendimento à Resolução CNE /CES nº 3, de 20 de junho de 2014, que atualizou as Diretrizes Curriculares Nacionais dos Cursos de Graduação em Medicina no Brasil e enfatizou a Atenção à Saúde, a Gestão em Saúde e a Educação em Saúde como as áreas fundamentais de formação do médico.

Em MIC I, os estudantes tiveram a oportunidade de conhecer o SUS, seus objetivos, princípios, diretrizes e atuação, de modo a ampliar sua visão acerca do sistema de saúde pública do País - do qual muitos, no futuro, farão parte na condição de profissionais. No módulo de MST I, o objetivo estava centrado na compreensão dos processos de trabalho no SUS e na abordagem de demandas específicas do ponto de vista da humanização, dos direitos humanos e da assistência qualificada para os segmentos populacionais objetos das políticas afirmativas de saúde étnico-raciais e de gênero.

Dialogando com a proposta das matérias, Muniz et al. ${ }^{12}$ afirmam que formar profissionais aptos a trabalharem de acordo com o que é proposto pelo SUS é tarefa difícil, e essa dificuldade é um reflexo do modelo de formação, hospitalocêntrico e fragmentado, baseado numa metodologia de ensino não problematizadora - ainda muito presente nos currículos dos cursos médicos País afora. Os autores defendem que, para a consolidação de um SUS de qualidade, tal como preconizado nas diretrizes que o regulamentam, o conhecimento de sua estrutura e principalmente de sua filosofia é a principal ferramenta para a efetivação e o alcance desses propósitos.

Em MIC I e MST I, o processo de inserção no trabalho das equipes da Estratégia de Saúde da Família e da atenção básica à saúde se deu de maneira suave e gradativa, iniciando-se com o reconhecimento interno e externo das áreas de cobertura de cada unidade, para que fosse possível a observação do perfil sociodemográfico das populações atendidas e sua contextualização no território. Os acadêmicos fizeram a territorialização e a contextualização de conhecimentos por meio do contato com as comunidades atendidas pelas UBS, seus usuários e profissionais, além de participarem de vivências em espaços específicos de promoção do cuidado em saúde.

Utilizando-se principalmente fundamentos advindos da Saúde Coletiva e das ciências humanas e sociais, as experiências pedagógicas proporcionadas foram capazes de levar os alunos a entenderem, na prática, o funcionamento do SUS e as dimensões políticas e administrativas da atenção básica. Por meio do contato com os serviços, do acompanhamento do trabalho dos agentes comunitários de saúde, da realização de entrevistas com os usuários, da análise da situação epidemiológica dos agravos nas comunidades e das visitas domiciliares desenvolvidas, foi possível conhecer in loco a realidade sociocultural dos usuários e do local em que estes estavam situados, estabelecendo uma conexão entre saberes acadêmicos, práticas de saúde e a percepção comunitária.

Por intermédio das atividades, viabilizou-se, ainda, a inserção preliminar dos acadêmicos em práticas de promoção e prevenção à saúde, por meio da participação em ações de programas existentes nas próprias instituições, como o programa de acompanhamento à gestante e o programa de controle de hipertensão e diabetes, além de atividades de puericultura, visitas domiciliares, acompanhamento de atendimentos, participação em ações de planejamento familiar e de educação em saúde e realização de outras atividades em diferentes espaços comunitários.

Sobre a presença do estudante no contexto da assistência à saúde, Caldeira et al. ${ }^{6}$ afirmam que se começa a criar um ambiente de intercâmbio de saberes e práticas entre os profissionais da equipe e há uma melhora em qualidade no funcionamento do serviço, uma vez que a presença do estudante pressiona os profissionais à prática reflexiva, reorientando suas práticas e aumentando a acessibilidade com a diversificação das atividades e horários de atendimento. Ainda segundo os autores (p. 5):

A presença do estudante de Medicina pode ser um fator propulsor da prática reflexiva dos profissionais, entendida como a habilidade de refletir criticamente sobre seu próprio raciocínio e decisões. Isto porque motiva o profissional a "executar" bem suas atividades e suscita dúvidas, fazendo com que ele reveja seus conhecimentos, estude e "pense alto". Estas ações 
diminuem o automatismo da prática e impulsionam o raciocínio analítico e a capacitação permanente.

Analisando um cenário educativo semelhante ao que aqui está sendo relatado, no qual houve a inserção dos estudantes em período inicial do curso universitário em locais de atenção básica, Gil et al. ${ }^{4}$ e Melo et al. ${ }^{13}$ constataram que, quando há integração entre os objetivos educacionais e o serviço, todos são beneficiados, inclusive a comunidade. Ao avaliarem essa mesma estratégia, Almeida et al. ${ }^{14}$, concluíram que a inserção do estudante de Medicina na atenção básica apresenta uma contribuição positiva para a academia e para a sociedade, visto que, com essa inserção comunitária, os estudantes tornam-se conhecedores e potenciais modificadores da realidade futura de seus locais de trabalho. Com esse mecanismo, sugere-se um atuar na formação profissional lastreado por reflexões críticas e socialmente referenciadas, de modo a tornar possível o desenvolvimento de ações de prevenção, promoção e educação em saúde efetivamente comprometidas com a realidade social contatada.

Em MIC I e MST I, a integração de aspectos teóricos e práticos permitiu reconhecer a importância da atuação integrada dos diferentes profissionais, além de visualizar o funcionamento dos serviços, sua organização e as sequências e processos relacionados ao atendimento das demandas de saúde da comunidade. Tais questões são apontadas por Mello et al. ${ }^{7}$ como essenciais para melhorar a interação profissional, o trabalho em equipe e para facilitar a atuação das equipes de saúde. Melo et al. ${ }^{13}$ concluíram que o convívio e a observação da rotina de trabalho e das atividades desenvolvidas pelos diferentes profissionais das UBS promove uma predisposição ao trabalho em equipe nos estudantes por permitir uma visão integrada do processo de trabalho na saúde, além do reconhecimento da importância, das limitações e das funções de cada especialidade.

O cenário da atenção básica se caracterizou, segundo mencionado por Caldeira et al. ${ }^{6}$, como um verdadeiro ambiente fértil para o desenvolvimento de diversas habilidades necessárias ao profissional de saúde. As atividades desenvolvidas nesses locais estão, ainda, em forte sintonia com o que preconizam as Diretrizes Curriculares Nacionais do Curso de Graduação em Medicina, em que a atenção integral à saúde e a integração ensino-serviço são enquadradas como aspectos basilares e que devem nortear a formação dos profissionais médicos ${ }^{15}$

A experiência possibilitou também uma reflexão crítica acerca do ambiente em que se vive e da sua interferência na qualidade de vida do indivíduo, da família e da comunidade.
Dessa forma, a inserção do aluno no contexto de adscrições territoriais possibilita que ele reconheça as dinâmicas de relacionamentos nos distintos cenários, trazendo à tona a capacidade de correlação entre os condicionantes e determinantes de saúde e o processo saúde-doença. Tal construção do conhecimento encontra-se embasada na magnitude de intervenção, nos vários níveis de prevenção, como recurso de amplificação das ações voltadas à promoção da saúde, em consonância com a Lei 8.080/199016, em seu artigo 3º, que dispõe:

Artigo 3: A saúde tem como fatores determinantes e condicionantes, entre outros, a alimentação, a moradia, o saneamento básico, o meio ambiente, o trabalho, a renda, a educação, o transporte, o lazer e o acesso aos bens e serviços essenciais; os níveis de saúde da população expressam a organização social e econômica do País.

Diante das diversas atividades desenvolvidas, podem-se fazer múltiplas reflexões acerca dos conceitos de saúde e doença. A partir da inserção dos discentes em vivências práticas na comunidade, pode-se, também, analisar e discutir relatos dos usuários do SUS, sobretudo os que se enquadram em grupos prioritários de atenção à saúde, com vistas ao desenvolvimento de competências para a compreensão de que o adoecimento decorre de um processo multidimensional e que este deve ser analisado sob a perspectiva do princípio da integralidade, previsto na Lei Orgânica da Saúde ${ }^{16}$.

A avaliação da última unidade do módulo de MIC I consistiu na elaboração de um plano de intervenção para uma das UBS cujos estudantes estabeleceram suas atividades práticas. Os alunos foram reunidos em cinco grupos, e, ao findar o módulo, realizou-se um ciclo de apresentações dos respectivos projetos de aplicabilidade local. Assim, três grupos elaboraram planos de intervenção cujos eixos temáticos residiam no fortalecimento do controle social e na estratégia de defesa dos interesses do bairro e da população local usuária atendida pelas unidades. Outros dois projetos tiveram como vertente o desenvolvimento de ações de educação em saúde para a prevenção de agravos prevalentes nas comunidades, que foi objeto de discussão de uma das atividades ao longo das disciplinas.

Em consonância com os achados de Pacheco ${ }^{17}$, a elaboração dos planos de intervenção evidencia que a universidade detém um papel histórico consolidado de produção de conhecimentos e oferta de subsídios que pautem as reais necessidades sociais. Dessa forma, traduz a apropriação pelos acadêmicos de Medicina de que as vivências práticas exercem significativas interferências no reconhecimento da importância da promoção à saúde, resgatando o olhar para os determinantes e 
condicionantes do processo saúde-doença. E, para além disso, desmistificam o paradigma de que o indivíduo deve recorrer aos serviços de saúde apenas quando necessita tratar agravos e enfermidades. Tais apontamentos apresentam forte correlação com Lima et al. ${ }^{5}$, que ressaltam a expansão das potencialidades das ações e serviços que constituem o SUS para além da ausência de doença, alcançando ativamente o completo estado de bem-estar.

A estruturação de planos de intervenção direcionados ao estímulo da cidadania dos usuários por meio do controle social expressa o reconhecimento, pelos estudantes, da importância da participação popular na formulação e no controle e execução de estratégias de saúde no âmbito territorial, alinhando-se aos Pactos pela Saúde, ao reordenamento do processo de trabalho de forma participativa e à ampliação dos programas horizontalizados com vistas à integralidade do cuidado, uma estratégia alternativa de contato com os usuários (gestores) do SUS².

Na atividade de conclusão da disciplina de MST I, os alunos, divididos em equipes, produziram documentários temáticos sobre os conteúdos abordados no módulo de "Políticas complementares e de educação popular em saúde". Esses documentários foram desenvolvidos a partir de uma série de visitas a espaços de promoção de cuidado em saúde e versaram sobre os saberes tradicionais, a educação popular em saúde e as políticas de atenção integral, com ênfase na saúde dos povos de terreiro de matriz afro-brasileira, na saúde dos povos indígenas, da comunidade LGBTT e da população do campo, da floresta e das águas. Tal proposta traz à tona a intenção de Azevedo $^{18}$ de reformular o ensino médico nos diversos aspectos metodológicos, passando a incluir as problemáticas de grupos prioritários, minorias e morbidades, entre outros, como a essência do aprendizado, a partir da interação com a comunidade, com o predomínio de atividades práticas sobre as teóricas, do trabalho em equipe e da maior aproximação com as ciências humanas e sociais.

A inserção dos alunos nas diversas problemáticas de saúde local durante as atividades desenvolvidas na disciplina de MST I culminou com uma avaliação coletiva. As contribuições identificadas foram relatadas pela maioria significativa do corpo discente, sendo os aspectos mais ressaltados pelos alunos o trabalho com a diversidade e o pluralismo de ideias, a ampliação das discussões sobre invisibilidade e marginalização dos grupos minoritários e a constituição de uma visão ampliada acerca da importância do diálogo entre os conhecimentos técnicos e saberes tradicionais. Durante o processo avaliativo, também foram identificadas várias narrativas que valorizaram a missão do papel social dos profissionais de saúde, principalmente sob as óticas da multidisciplinaridade e integralidade. Esses resultados revelam um caráter participativo no replanejamento dos métodos propostos na disciplina, frente à percepção da realidade dos discentes e da busca de um processo de ensino-aprendizagem capaz de construir a essência do trabalho em saúde coletiva ${ }^{19}$.

Outros objetivos também relevantes, presentes durante a realização das atividades de ambas as matérias, foram a troca de saberes e as interações socioculturais com as comunidades e o contato com os mais diversos conceitos de saúde absorvidos nos diferentes contextos e situações experimentadas, fundamentais à construção de uma perspectiva ampliada sobre o cuidado partir da ótica do novo paradigma do processo de saúde-doença e do pensamento complexo. Em conformidade com Araújo et al. ${ }^{20}$, tais vivências permitiram a aplicação prática dos conhecimentos teóricos problematizados em sala de aula, uma vez que o contato precoce do aluno de Medicina com a realidade da saúde pública remete ao seu papel de permanente agente efetivo da atenção à saúde junto à sociedade.

O período de realização das atividades proporcionou experiências ímpares para toda a formação médica. Por meio das disciplinas referidas, além das contribuições já relatadas, trabalhou-se o aperfeiçoamento do relacionamento interpessoal no contato tanto com o paciente quanto com os profissionais e o desenvolvimento de habilidades de comunicação, acolhimento e humanização como inovações capazes de mudar o paradigma que orienta o ensino médico, redirecionar as práticas profissionais em saúde e servir como alavancas dos processos de mudança no ensino da Medicina ${ }^{4}$.

\section{CONCLUSÕES}

Os maiores legados dos eixos MIC I e MST I foram permitir ao discente do curso médico: (i) o desenvolvimento da capacidade de entender o paciente como um indivíduo dotado de influências ambientais e características que ultrapassam os aspectos biológicos; (ii) o estímulo à reflexão acerca da relevância de considerar aspectos culturais, sócio-históricos e políticos como elementos para a desconstrução dos preconceitos e processos discriminatórios para o reconhecimento da alteridade e da afirmação das diferenças, com base na equidade; (iii) a reflexão acerca da importância da aplicação desse conceito no âmbito do SUS; (iv) e ter possibilitado o preparo e o desenvolvimento de habilidades necessárias à atuação na atenção básica e no SUS de forma crítico-reflexiva e em equipes multidisciplinares, com a consequente valorização de cada profissional e das mais distintas especialidades em saúde.

Os métodos de aprendizagem proporcionados pelos módulos disciplinares permitiram ao aluno de Medicina a oportunidade de lidar, desde o início de sua formação, com 
o cotidiano de uma comunidade e de diferentes contextos de saúde. Confrontar aspectos teórico-práticos proporcionou uma aprendizagem adquirida por meio de experiências e da integração ensino-serviço, viabilizando a contextualização de ensinamentos no cenário comunitário e social.

As atividades configuraram experiências altamente enriquecedoras e espaços privilegiados de formação acadêmica e cidadã para o futuro profissional médico, além de ferramentas efetivas para a aplicação do que as DCN preconizam para uma formação alinhada às necessidades da comunidade e do SUS. Dessa forma, as cadeiras se mostraram espaços eficientes na busca de oferta de melhores condições de saúde aos usuários do SUS e de maior resolutividade de suas demandas.

\section{REFERÊNCIAS}

1. Azevedo BMS, Ferigato S, Souza TP, Carvalho SR. A formação médica em debate: perspectivas a partir do encontro entre instituição de ensino e rede pública de saúde. Interface (Botucatu) [Internet]. 2013 Mar; 17(44):187-200. DOI: 10.1590/S1414-32832012005000048.

2. Batista KBC, Gonçalves OSJ. Formação dos profissionais de saúde para o SUS: significado e cuidado. Saúde soc. [Internet]. 2011 Dez; 20(4):884-899. DOI: 10.1590/S010412902011000400007.

3. Franco CAGS, Cubas MR, Franco RS. Currículo de medicina e as competências propostas pelas diretrizes curriculares. Rev. Bras. de Educ. Médica. 2014; 38(2):221-230. DOI: 10.1590/S0100-55022014000200009.

4. Gil CRR, Turini B, Cabrera MAS, Kohatsu M, Orquiza SMC. Interação ensino, serviços e comunidade: desafios e perspectivas de uma experiência de ensino-aprendizagem na atenção básica. Rev. Bras. Educ. Med. 2008; 32(2):230-9. DOI: 10.1590/S0100-55022008000200011

5. Lima LJ, Assis RRQ, Lima Júnior JF. Formação de recursos humanos e promoção da saúde: uma análise das diretrizes curriculares nacionais para a graduação em medicina. Revista de Pesquisa Interdisciplinar 2017 Set; 2(2):327-345. DOI: 10.24219/rpi.v2i2.285.

6. Caldeira ES, Leite MTS, Rodrigues-Neto JF. Estudantes de Medicina nos serviços de atenção primária: percepção dos profissionais. Rev. Bras. Educ. Med. 2011;35(4):477-485. DOI: $10.1590 /$ S010055022011000400006.

7. Mello CCB, Alves RO, Lemos SMA. Metodologias de ensino e formação na área da saúde: revisão de literatura. Rev. CEFAC 2014.2014; 16(6): 2015-2028. DOI: 10.1590/19820216201416012.

8. Gomes R, Brino RF, Aquilante AG, Avó LRS. Aprendizagem Baseada em Problemas na formação médica e o cur- rículo tradicional de Medicina: uma revisão bibliográfica. Rev. Bras. Educ. Med. 2009, 33(3): 433-440. DOI: 10.1590/ S0100-55022009000300014.

9. Sugestões para os relatos de experiência profissionais. Periódicos Eletrônicos de Psicologia. 2014. Disponível em: http:/ / pepsic.bvsalud.org. Acesso em: 10 jul. 2017.

10. Gil AC. Como elaborar projetos de pesquisa, São Paulo: Atlas; 2008.

11. Kemmis, Mc Taggart (1988) apud Tripp, D. Pesquisa-ação: uma introdução metodológica. Educação e Pesquisa 2005;31(3):443-466. DOI:10.1590/S1517-97022005000300009.

12. Muniz LDRC, Costa LA, Muniz VRC. Formação de profissionais para o SUS: a importância da educação permanente em saúde. In: X Simpósio de Produção Científica da UESPI: Anais do X Simpósio...; dez 2010; Teresina: UESPI; 2011.

13. Melo BT, Jordão DA, Beserra KS, Andrade LSGM, Lima Junior RCC, Batista RSL, Cavalcante MT. Uma experiência de integração ensino, serviço e comunidade de alunos do curso de graduação em medicina na atenção básica no município de Maceió-AL, Brasil. Revista Ciência Plural. 2017; 3(3):69-80.

14. Almeida FCM, Maciel APP, Bastos AR et al. Avaliação da inserção do estudante na Unidade Básica de Saúde: visão do usuário. Rev. Bras. Educ. Med. 2012; 36(1):33-39. DOI: 10.1590/S0100-55022012000200005.

15. Ministério da Educação (BR), Conselho Nacional de Educação. Resolução nº 3, de 20 de junho de 2014. Institui Diretrizes Curriculares Nacionais do Curso de Graduação em Medicina. Diário Oficial da União. Brasília, 23 jun. 2014; 117(1): 8-11.

16. Brasil. Presidência da República. Lei 8.080 de 19 de setembro de 1990. Dispõe sobre as condições para a promoção, proteção e recuperação da saúde, a organização e o funcionamento dos serviços correspondentes e dá outras providências. Diário Oficial da União. Brasília, 20 set. 1990; Seção 1.

17. Pacheco EN, Freire RC. Integração ensino-serviço-comunidade nos projetos de cooperação do internato de Medicina Social da Faculdade de Medicina da Bahia/Universidade Federal da Bahia: operacionalização de conceitos. Monografia. Salvador: 2014, 53 p.

18. Azevedo GD, Vilar MJP. Educação médica e integralidade: o real desafio para a profissão médica. Rev. Bras. Reumatol; 46(6): 407-409, nov-dez 2006. DOI: 10.1590/S048250042006000600008

19. Sá PPT, Odeh MM. Perfil da saúde coletiva dentro da disciplina introdução a práticas de saúde: relato de experiência. 
Revista Tempus: Actas de Saúde Coletiva, 7(3): 129-133, jan-jun2017. DOI: 10.18569/tempus.v7i3.1399.

20. Araújo GB, Alves Filho FWP, Santos RS, Lira RCM. Territorialização em saúde como instrumento de formação para estudantes de medicina: relato de experiência. Revista de Políticas Públicas; 16(1): 124-129, jan-jun2017.

\section{CONTRIBUIÇÃO DOS AUTORES}

Claudio José dos Santos Júnior participou da concepção e delineamento do trabalho, da sistematização, análise, interpretação e discussão dos dados e da revisão final do artigo.

Jailton Rocha Misael participou da escrita e revisão do artigo. Maria Rosa da Silva participou co-orientação do estudo e da revisão da versão final do artigo.

Valmir de Melo Gomes participou do delineamento, coordenação e orientação do estudo e da revisão da versão final do artigo.

\section{CONFLITO DE INTERESSES}

Não há conflito de interesses.

\section{ENDEREÇO PARA CORRESPONDÊNCIA}

Claudio José dos Santos Júnior. Faculdade de Medicina da UNCISAL (FAMED - UNCISAL). Rua Dr. Jorge de Lima, 113, Trapiche da Barra. CEP: 57010-300. Maceió-AL. E-mail: claudiosantos_al@hotmail.com. 